\title{
SCA17 caused by homozygous repeat expansion in TBP due to partial isodisomy 6
}

\author{
CH Zühlke*,1, M Spranger ${ }^{2}$, S Spranger $^{3}$, R Voigt $^{1}$, M Lanz $^{4}$, U Gehlken ${ }^{1}$, F Hinrichs ${ }^{1}$ \\ and E Schwinger ${ }^{1}$
}

${ }^{1}$ Institut für Humangenetik, Universität Lübeck, 23538 Lübeck, Germany; ${ }^{2}$ Neurologisches Rehabilitationszentrum Friedehorst, 28717 Bremen, Germany; ${ }^{3}$ Praxis für Humangenetik, 28205 Bremen, Germany; ${ }^{4}$ Neurologische Klinik, Zentralkrankenhaus Bremen Ost, 28325 Bremen, Germany

An expanded polyglutamine domain in the TATA-binding protein (TBP) has been described in patients with spinocerebellar ataxia type 17 (SCA17) characterized by cerebellar ataxia associated with dementia. TBP is a general transcription initiation factor that regulates the expression of most eukaryotic genes transcribed by RNA polymerase II. SCA17, as an autosomal dominantly inherited progressive neurodegenerative disorder, is caused by heterozygous expansion of a CAG repeat coding for glutamine. Alleles with $\mathbf{2 7}$ to a maximum of $\mathbf{4 4}$ glutamine residues were found as the normal range, whereas expansions above 45 repeat units were considered pathological. Here, we present a patient with a very severe phenotype with a late onset but rapidly progressing ataxia associated with dementia and homozygous $\mathbf{4 7}$ glutamine residues caused by an apparent partial isodisomy 6 . This extraordinary case has important implications for the insights of TBP and SCA17. The expanded polyglutamine domain in both TBP copies is not correlated with embryonic death indicating that the normal function of the protein is not disrupted by this kind of mutation but may account for the dementia seen in this patient.

European Journal of Human Genetics (2003) 11, 629-632. doi:10.1038/sj.ejhg.5201018

Keywords: SCA17; CAG repeat expansion; TATA-binding protein; partial isodisomy

\section{Introduction}

Unstable expansions of trinucleotide repeats have been shown to cause at least 14 neurological diseases ${ }^{1}$ including six spinocerebellar ataxias (SCAs). SCAs are autosomal dominantly inherited neurodegenerative disorders with a predominantly adult age of onset, clinical and genetic heterogeneity, and expanded polyglutamine domains. Recently, this kind of mutation was detected within the TATA-binding protein (TBP) gene (MIM 600075), too, producing a SCA-like phenotype. The gene for the TBP, a general transcription initiation factor that regulates the expression of most eukaryotic genes transcribed by RNA

*Correspondence: Dr CH Zühlke, Institut für Humangenetik, Universität Lübeck, Ratzeburger Allee 160, 23538 Lübeck, Germany. Tel: +49 451500 2622; Fax: +49 451500 4187;

E-mail: zuehlke@medinf.mu-luebeck.de

Received 25 February 2003; revised 12 March 2003; accepted 21 March 2003 polymerase $\mathrm{II}^{2-4}$ is located at chromosome $6 \mathrm{q} 27^{5}$ and includes a highly polymorphic imperfect repeat. ${ }^{6}$ This polyglutamine-encoding DNA sequence can be divided into five regions including two polymorphic $(\mathrm{CAG})_{n}$ stretches: $(\mathrm{CAG})_{3}(\mathrm{CAA})_{3} \cdot(\mathrm{CAG})_{n} \cdot \mathrm{CAA}$ CAG CAA $\cdot(\mathrm{CAG})_{\mathrm{n}}$. CAA CAG. Gostout et $a l^{7}$ found 20 different alleles with 29-40 glutamine residues in the aminoterminal region of the wild-type protein.

Assuming a further association between repeat expansions and neurological diseases, the TBP gene has been investigated intensively as a candidate locus. Among patients with various forms of neurological diseases, one individual showed a de novo expansion of the repeat in the TBP gene because of a partial duplication ${ }^{8}$ resulting in an expanded polyglutamine domain with 63 amino-acid residues. Clinically, this patient presented with ataxia, short stature, atypical absences, pyramidal signs, and mental deterioration. The symptoms started during childhood. 
Recently, we presented the first familial cases with expanded polyglutamine stretches in the TBP gene. ${ }^{9}$ While normal alleles in the German population contain up to a maximum of 44, pathological alleles associated with clinical symptoms range between 50 and 55 glutamine residues. In addition, we found an allele coding for 48 glutamine residues that was stable inherited but did not cosegregate with the disease phenotype in six siblings. ${ }^{10}$ On the other hand, instability of expanded alleles during transmission could also be demonstrated in hereditary cases. ${ }^{9}$ Neurological symptoms in these patients were predominant cerebellar ataxia with variable involvement of pyramidal tracts and basal ganglia.

In contrast, an expanded allele with 46 repeat units has been shown to be stable during transmission in a Belgian family. ${ }^{11}$ The main clinical features were cerebellar ataxia, dementia, and behavioural disturbances with onset in the fourth to sixth decade. Alleles ranging from 47 to 55 repeats in Japanese patients with cerebellar ataxia and additional extracerebellar symptoms including dementia, pyramidal and basal ganglia symptoms were also found to be stable during transmission. ${ }^{12}$ Transmission occurred autosomal dominantly in three of the Japanese cases. The authors also detected neuronal intranuclear inclusions in the brain of an individual carrying 48 repeats. These intranuclear inclusions are characteristic findings in other types of SCA. Their presence therefore gives support to the hypothesis that expansions in the TPB protein gene should be causative for a new type of spinocerebellar ataxia, named SCA17. All patients described to date show repeat expansions only in one allele in addition to an allele within the normal range.

Looking for genetic factors for neurodegenerative diseases, we investigated a 40-year-old female patient with rapidly progressive cerebellar ataxia and dementia. Surprisingly, we found an apparent homozygosity for a glutamine repeat of 47 residues in the TBP, but no allele in the normal range.

\section{Materials and methods Clinical data of the patient}

A 40-year-old female patient with rapidly progressive cerebellar ataxia and dementia was investigated. Neurological problems had not been apparent during youth. She finished school without delay and started a university career, however did not finish with a degree. In the following years, she suffered from fluctuating depressions and progressive problems in the activities of daily living. She was increasingly handicapped by progressive ataxia for the last 3 years. On admission, she was disorientated, completely neglected, and unable to care for herself.

Neurological examination showed saccadic eye movements, cerebellar dysarthria, brisk deep tendon reflexes with flexor plantar response, an ataxic gait and intention tremor, although no pareses or deficits in sensitivity. MRI showed cerebellar atrophy without any other pathology. Electromyography, electroneurography, and analysis of cerebrospinal fluids gave normal results.

Neurological information concerning her parents who are deceased is not available. Consanguinity or further siblings are not known.

\section{Genotyping and mutation analysis}

For molecular analyses, total genomic DNA was extracted from peripheral blood leucocytes by standard protocols.. Routine molecular diagnostic for FRDA, SCA1, 2, 3, 6, 7, 10, and 12 loci revealed heterozygosity for two normal-sized alleles. For SCA17, PCR was performed using primers described by Koide et al. ${ }^{8}$ Products were separated and visualized on $6 \%$ denaturing polyacrylamide gels and silver staining.

To determine the nucleotide sequence of SCA17 alleles, PCR products were cloned into the vector pCR 2.1-TOPO (Invitrogen) and sequenced using the dideoxy chain termination method. In all, 10 single colonies have been analysed.

To look for deletions, 16 highly polymorphic markers drawn from Genome Database and covering the entire chromosome 6 have been analysed (Table 1). Microsatellite marker alleles were amplified by PCR and resolved on a $6 \%$ denaturing polyacrylamide gel.

\section{Cytogenetic analysis of the patient}

For high-resolution chromosomal analysis, peripheral blood lymphocyte cultures were stimulated by phythaemagglutinin (PHA) and synchronized by methotrexate using standard protocols. Metaphase chromosomes were routinely analysed by standard Trypsin-Giemsa banding.

Table 1 Typing with polymorphic markers derived from $\mathrm{NCBI}$ chromosome 6 map

\begin{tabular}{lccc}
\hline Marker & Alleles & kbp position & Chrom. position \\
\hline D6-F13A1 & $1-5$ & 12,710 & $\mathrm{p} 24$ \\
SCA1 & $30-33$ & 16,357 & $\mathrm{p} 23$ \\
D6S2439 & $1-2$ & Unknown & $\mathrm{p} 21$ \\
D6S1017 & $2-3$ & 48,097 & $\mathrm{p} 21$ \\
D6S1053 & $4-6$ & 75,386 & $\mathrm{q} 13$ \\
D6S1056 & $4-6$ & $1,05,934$ & $\mathrm{q} 16$ \\
D6S474 & $1-3$ & $1,24,891$ & $\mathrm{q} 22$ \\
D6S1009 & $4-5$ & $1,49,433$ & $\mathrm{q} 23$ \\
D6S-B & $1-10$ & $1,60,098$ & $\mathrm{q} 24$ \\
D6S305 & 14 & $1,74,246$ & $\mathrm{q} 25$ \\
D6S1277 & 4 & $1,76,349$ & $\mathrm{q} 26$ \\
D6S1719 & 4 & $1,78,121$ & $\mathrm{q} 27$ \\
D6S264 & 1 & $1,78,760$ & $\mathrm{q} 27$ \\
D6S281 & 2 & $1,81,809$ & $\mathrm{q} 27$ \\
D6S446 & 2 & $1,82,365$ & $\mathrm{q} 27$ \\
D6S1590 & 4 & $1,82,573$ & $\mathrm{q} 27$ \\
TBP & 47 & $1,82,684$ & $\mathrm{q} 27$ \\
\end{tabular}


In addition, to investigate deletions hybridization with the subtelomeric probe 6qter; D6S522 was performed in 50 mitoses as well as fluorescence in situ hybridization with YAC probes specific for chromosome 6q24-27 (Y802b09: 6q24;D6S1648, Y804d08: 6q25;D6S290/D6S440, Y911c10: 6q27;D6S1697/D6S297, Y933f07: 6q27;D6S281/6WI-8780) kindly provided by the Max-Planck Institute for Molecular Genetics, Berlin.

\section{Results and discussion}

Investigating a 40-year-old female patient with rapidly progressive cerebellar ataxia and dementia, we found heterozygosity for two normal-sized alleles for FRDA, SCA1, 2, 3, 6, 7, 10, and 12 loci, but an apparent homozygosity for an expanded allele in the TBP and no allele in the normal range. The following sequence analyses revealed just one SCA17 allele with the triplet composition $(\mathrm{CAG})_{3} \quad(\mathrm{CAA})_{3} \quad(\mathrm{CAG})_{9}$ CAA CAG CAA $(\mathrm{CAG})_{27}$ CAA CAG coding for 47 glutamine residues.

Genotyping with microsatellites including the SCA1 repeat revealed heterozygosity for nine loci between $6 \mathrm{p}$ and 6q24, but only one allele for eight markers including the SCA17 repeat between 6q25 and 6q27 (Table 1). This result may be explained by a partial deletion, consanguinity of the parents, or a partial uniparental isodisomy at chromosome 6q. Unfortunately, no relatives of the patient are available for molecular testing. To examine the deletion hypothesis, cytogenetic analyses have been performed. Conventional karyotyping showed normal chromosomes in the patient $(46, \mathrm{XX})$ without structural aberrations (resolution 500 bands following ISCN). Induced by the location of the TBP gene near the telomeric region on chromosome 6q, hybridization with the subtelomeric probe 6qter; D6S522 was performed but detected no deletion in 50 mitoses. Furthermore, fluorescence in situ hybridization with YAC probes specific for chromosome 6q24-27 revealed two signals in all metaphases. Therefore, a deletion in the long arm of chromosome 6 is very unlikely and the missing heterozygosity of polymorphic markers at $6 \mathrm{q}$ is the result of partial isodisomy 6 caused by consanguinity of the parents or from uniparental origin. No DNA of the patient's parents is available to differentiate these two hypotheses. However, in any case the patient would be homozygous for the repeat expansion. But, is the pure severe neurological phenotype compatible with a partial isodisomy?

In contrast to UPD 7 (Silver-Russell syndrome) or UPD 15 (Prader-Willi and Angelman syndrome), UPD 6 is in general not correlated with a significant severe phenotype, but has been described in patients with (transient) neonatal diabetes mellitus or intrauterine growth retardation. ${ }^{13}$ Remarkably, the partial isodisomy observed in our patient and explained by mitotic recombination events ${ }^{13}$ causes a very progressive neurological disease.
Severe phenotypes in neurodegenerative disorders may be linked with homozygous repeat expansions, for example, an earlier age of onset and a more severe clinical manifestation were shown for a patient homozygous for the SCA6 repeat expansion, ${ }^{14}$ although variability in disease progression is documented as well in those cases. ${ }^{15}$ Furthermore, intermediate SCA6 alleles in homozygous condition may produce pathogenic effects. ${ }^{16}$ Unfortunately, we could not investigate if the $(\mathrm{Glu})_{47}$ allele detected in the patient presented here is associated with reduced penetrance as shown for a $(\mathrm{Glu})_{48}$ allele in one of our families. ${ }^{10}$

More severe disease phenotypes in patients homozygous for expanded alleles were found also in SCA3 $3^{17,18}$ in contrast to Huntington's disease (HD). Homozygotes for the HD mutation do not differ in clinical expression from typical heterozygotes. ${ }^{19}$ In general, the neurodegeneration occurring in CAG repeat diseases is considered to share a common mechanism resulting in a gain of toxic functions related to the expanded polyglutamine tracts without disruption of the wild-type function. This model would be confirmed for the TBP by the patient presented here since the total loss of normal TBP function is not compatible with life. ${ }^{20}$ This case shows that the level of specificity and activity of a TBP with 47 glutamine residues is sufficient for the development of a human organism. But, it is not excluded that partial loss of TBP function may occur and account for dementia in patients as described here. However, the phenotypic expression in individuals homozygous for translated CAG repeat 9 expansions is sometimes controversial and points to dose-dependent additional pathogenic effects.

\section{Acknowledgements}

We would like to thank A Dalski, Y Hellenbroich and S Opitz for helpful discussions. YAC probes specific for chromosome 6q24-27 were kindly provided by the Max-Planck Institute for Molecular Genetics, Berlin.

\section{References}

1 Cummings CJ, Zoghbi HY: Fourteen and counting: unraveling trinucleotide repeat diseases. Hum Mol Genet 2000; 9: 909-916.

2 Hoffmann A, Sinn E, Yamamoto T et al: Highly conserved core domain and unique $\mathrm{N}$ terminus with presumptive regulatory motifs in a human TATA factor (TFIID). Nature 1990; 346: 387-390.

3 Kao CC, Lieberman PM, Schmidt MC et al: Cloning of a transcriptionally active human TATA binding factor. Science 1990; 248: 1646-1650

4 Peterson MG, Tanese N, Pugh BF, Tjian R: Functional domains and upstream activation properties of cloned human TATA binding protein. Science 1990; 248: 1625-1630.

5 Imbert G, Trottier Y, Beckmann J, Mandel JL: The gene for the TATA binding protein (TBP) that contains a highly polymorphic protein coding CAG repeat maps to 6q27. Genomics 1994; 21 : 667-668.

6 Polymeropoulos MH, Rath DS, Xiao H, Merril CR: Trinucleotide repeat polymorphism at the human transcription factor IID gene. Nucleic Acids Res 1991; 19: 4307. 
7 Gostout B, Liu Q, Sommer SS: Cryptic repeating triplets of purines and pyrimidines (cRRY(i)) are frequent and polymorphic: analysis of coding cRRY(i) in the proopiomelanocortin (POMC) and TATAbinding protein (TBP) genes. Am J Hum Genet 1993; 52: 1182-1190.

8 Koide R, Kobayashi S, Shimohata T et al: A neurological disease caused by an expanded CAG trinucleotide repeat in the TATAbinding protein gene: a new polyglutamine disease? Hum Mol Genet 1999; 8: 2047-2053.

9 Zühlke C, Hellenbroich Y, Dalski A et al: Different types of repeat expansion in the TATA-binding protein gene are associated with a new form of inherited ataxia. Eur J Hum Genet 2001; 9: 160-164.

10 Zühlke Ch, Gehlken U, Hellenbroich Y et al: Phenotypical variability of expanded alleles in the TATA-binding protein gene: reduced penetrance in SCA17? J Neurol 2003; 250: 161-163.

11 Fujigasaki H, Martin J-J, De Deyn PP et al: CAG repeat expansion in the TATA box-binding protein gene causes autosomal dominant cerebellar ataxia. Brain 2001; 124: 1939-1947.

12 Nakamura K, Jeong S-Y, Uchihara T et al: SCA17, a novel autosomal dominant cerebellar ataxia caused by an expanded polyglutamine in TATA-binding protein. Hum Mol Genet 2001; 10: 1441-1448.

13 Eggermann T, Marg W, Mergenthaler S et al: Origin of uniparental disomy 6: presentation of a new case and review on the literature. Ann Génétique 2001; 44: 41-45.
14 Matsumara R, Futamara N, Fujimoto Y et al: Spinocerebellar ataxia type 6. Molecular and clinical features of 35 Japanese patients including one homozygous for the CAG repeat expansion. Neurology 1997; 49: 1238-1243.

15 Kato T, Tanaka F, Yamamoto $\mathrm{M}$ et al: Sisters homozygous for the spinocerebellar ataxia type 6 (SCA6)/CACNA1A gene associated with different clinical phenotypes. Clin Genet 2000; 58: 69-73.

16 Mariotti C, Gellera C, Grisoli M et al: Pathogenic effect of an intermediate-size SCA-6 allele (CAG)(19) in a homozygous patient. Neurology 2001; 57: 1502-1504.

17 Takiyama Y, Igarashi S, Rogaeva EA et al: Evidence for intergenerational instability in the CAG repeat in the MJD1 gene and for conserved haplotypes at flanking markers amongst Japanese and Caucasian subjects with Machado-Joseph disease. Hum Mol Genet 1995; 4: 1137-1146.

18 Lerer I, Merims D, Abeliovich D et al: Machado-Joseph disease: correlation between clinical features, the CAG repeat length and homozygosity of the mutation. Eur J Hum Genet 1996; 4: 3-7.

19 Wexler NS, Young AB, Tanzi RE et al: Homozygotes in Huntington's disease. Nature 1987; 326: 194-197.

20 Veenstra GJC, Weeks DL, Wolffe AP: Distinct roles for TBP and TBP-like factor in early embryonic gene transcription in Xenopus. Science 2000; 290: 2312-2314. 